\title{
Robotic Path Planning and Fuzzy Neural Networks
}

\author{
Nada Mirza \\ College of Engineering, Al Ain University, UAE
}

\begin{abstract}
Fuzzy logic has gained excessive attention due to its capacity of handling the data in a much simpler way. It is applied to decrease the intricacy of already existed solutions and to provide the solution of new problems also. On the other hand, neural networks are distinct because of their robust processing and adaptive capabilities in dynamic environment. This paper mainly reviews the primary ideas and contribution of neural network system and fuzzy logic in the field of robotic path planning. Several hybrid techniques, which are being utilized in bringing dream of mobile robots to reality are discussed.
\end{abstract}

Keywords: Neural networks, fuzzy logic, obstacle avoidance, path planning.

Received February 29, 2020; accepted June 9, 2020

https://doi.org/10.34028/iajit/17/4A/5

\section{Introduction}

In the past few years, the world is resorting to automating all areas of every day human life such as industries, hospitals, supermarkets, ... through the use of mobile robots in order to improve the performance and the accuracy of the work. These mobile robots must be able to react whether in known environment (structured), in semi-known environment or in unknown environment (unstructured). The navigation in unknown environment requires the use of reactive approaches with which the mobile robot will be able to react to any situation.

A forecast can be established in the favor of mobile robotic technology if we study the recent trends utilization of mobile robots. Now a day's mobile robots are being used everywhere [2, 11]. For example, they are being used in room cleaning, helping people in their jobs, automation companies etc. for all these applications they need to have the ability to plan their path and move freely like humans $[5,25]$.

To make sure the safe interaction between humans and robotic devices, various tracks of research are being carried out. Successful path planning means the movement of mobile robot in real environment without causing any kind of destruction and harm towards the human beings. To make this work robot should be able to avoid obstacles in its path. Obstacle avoidance can be further divided into two categories, motion control and dynamic control depending on various conditions. In the past few years many algorithms are developed to cater for this problem but memory and computational limitations were not considered. That's why recently advanced control methods are incorporated which are also known as hybrid algorithms. They combine the regular computational control methods with the neural network topologies [22, 24].

Collision avoidance $[4,6]$ is a common problem in robotics and many methods have been employed to solve it. The major challenges faced in the design of an intelligent robotic system is to plan the goal reaching tasks and then to plan the robot motion activities. Neuro-fuzzy networks present a solution which is different from classical models and heuristic approaches. Classical methods are computationally expensive when there is a complex problem. Reason behind excessive computational cost of classical technique is their derivation from graph searching method [20]. Most existing approaches that make use of neural networks tend to either only deal with scenarios with very few obstacles or with path following implementations where a direct path to the goal is always available. An obstacle avoidance task in an unknown environment is a very complex task. A way to deal with such type of complex task is to divide this complex task in to number of small subtasks.

This paper is extended version of paper published in 2019 International Arab Conference on Information Technology [12].

After introduction paper is divided into following sections. Section 2 provides brief introduction about mobile robots. Sections 3 and 4 are defining the Neural Network (NN) and fuzzy logic respectively. Combination of fuzzy logic with $\mathrm{NN}$ is discussed in section 5. Whereas section 6 is conclusion for the study being conducted in this paper.

\section{Mobile Robots}

Mobile robots are automated structure which are capable of moving on their own without any help. To make the mobile robots more useful and to replace human beings with them in the dangerous working fields, they need to mimic the behavior of human beings. For that reason, neural networks are involved in developing the algorithms which can help to control them. One of the very basic feature of mobile 
robot is to be able to move freely and plan a shortest path for the movement [27].

Intelligent Mobile Robot is one kind of robot systems which can sense environment and self-state by sensor, and achieve target-oriented independent movement in the obstacle condition and then complete specific task function. Mobile Robot Navigation is that the mobile robot can independently complete non-collision movement according to the environment information when it is in an unknown, complex dynamic change condition. At the same time, it reduces consumption (e.g. time and energy) as far as possible. Navigation is one of the base functions that mobile robot should have. Path planning is the key area of mobile robot navigation research now. It includes global path planning and local path planning. The main method of local path planning includes artificial potential field, fuzzy logic algorithm, genetic algorithm and so on.

Real time path planning of mobile robot means a collision free path from initial point to the destination [16]. There can be two kind of environment for this problem. One in which we can define the obstacles already. Other one is considered as dynamic environment in which out obstacles are moving. Designing an algorithm for the first case is relatively easy task but the problem arises when there are non-stationary obstacles. One of the very first algorithm developed to address this problem was in 1979 by Lozano-Perez-and Wesley, [10]. Starting from 1979 till now focus is remained on making path planning faster and more reliable. Typical computational methods were not enough to address this problem. As it needs to mimic human behavior to make decision at spot with respect to all the dynamic changes happening around.

\section{Neural Networks}

Artificial Neural Networks is a kind of network that simulates the human brain's thinking ability and realizes nonlinear algorithm function through a large number of simulated neurons. It is similar to the process of processing information in the human brain and has the learning and self-adaptive functions. The difference is that its theoretical basis is the mathematical network topology. It uses the neural network's own structure to express the input-output relationship, so that the network has a nonlinear mapping function. Perform parallel processing. The main features of artificial neural networks are non-linear networks capable of processing information in parallel, and they have learning functions, fault tolerance, distributed storage of information, and robustness. The learning rules of artificial neural networks mainly include error correction learning, competitive learning and Hebb learning and so on. Thus, Neural Network (NN) can be defined as an ability to mimic the human behavior. Research in this field is done due to various reasons. One of them if to make automated system more intelligent and useful. Basic architecture of $\mathrm{NN}$ is provided in Figure1.

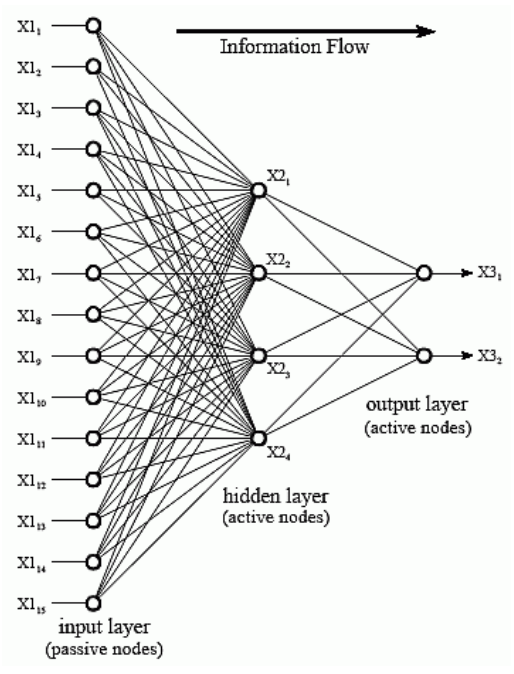

Figure 1. Neural network architecture [9].

From Figure 1 basic line of operation of neural network can be understood. Number of layers vary from system to system [19]. Generally speaking, for three-layer structure, layer 1 serve as input variables, layer 2 is considered as hidden layer member ship functions are implemented in layer 2. Whereas last layer is output layer.

\section{Fuzzy Theory}

History of fuzzy theory goes back to 1965 when it was first defined by Professor Lofti Zadeh. It refers to the theory that uses the basic concepts of fuzzy sets or continuous membership functions.

Fuzzy theory can be used to find the best path for robots using the strategy of angle deviation to the required position and distance measured from the obstacle in the desired route. [8, 17, 18].

Simple fuzzy reasoning block diagram is displayed in Figure 2.

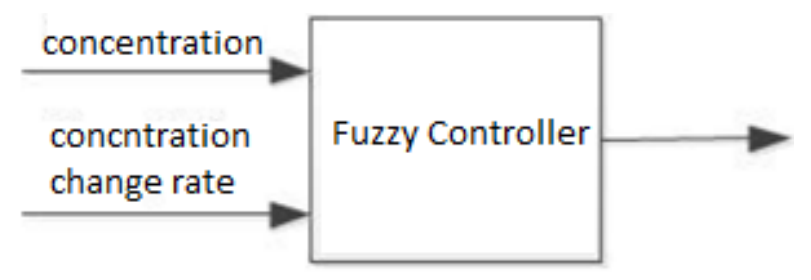

Figure 2. Fuzzy reasoning block diagram [21].

It changes the human language problems into the mathematical equations. It increases the simplicity and flexibility of the system.

It contains multiple if-then rule statements. It can be implemented in different size systems. Basic structure for developing a fuzzy system is given in Figure 3. 


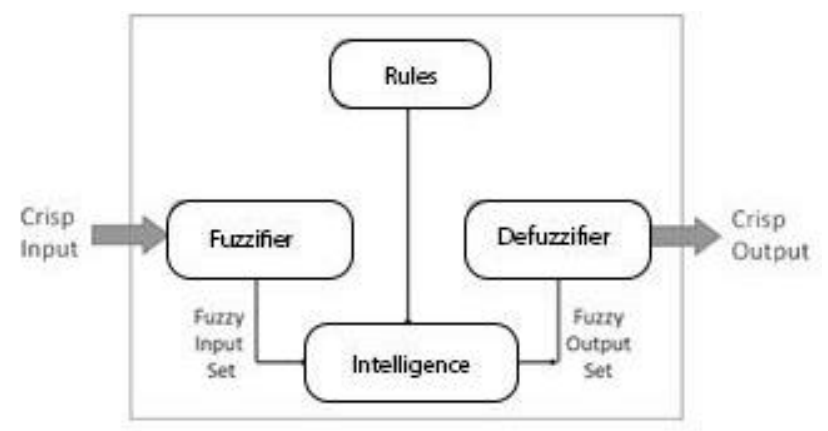

Figure 3. Fuzzy logic system architecture [14, 21].

It deals with the relationship between input and output of the system. It provides more precise, steady and vigorous performance, the simplicity in the system declines the complexity of design.

There are many applications being implemented with the help fuzzy systems. One of them is to bring the ultimate dream of technology into reality, that is to make human like automated structures. NN and fuzzy logic are integrated together to make the systems more reliable and intelligent $[7,14,15,21]$.

\section{Neuro-Fuzzy Controller}

The neural network and fuzzy logic are combined using certain means to form a new structure of technology, called neural-fuzzy technology. Their fusion mainly includes neural network and fuzzy system integration, fuzzy logic enhanced neural network and fuzzy neural network. By this manner learning and computational capabilities of neural network can be joined with the reasoning and representation of fuzzy logic systems [1, $7,13]$.

Through research, it has been found that the two technologies of fuzzy logic and neural network have their own strengths. Therefore, certain technologies are used to properly combine fuzzy logic and neural networks to form a new structure. This structure draws two strengths, and having their own characteristics on the basis of the original features, are better than a separate neural network or a separate fuzzy system. For a complex system such as path planning of mobile robots, the fuzzy neural control technology has great advantages.

These days it's a common practice to add fuzzy logic in the NN to robust the system. The combined FuzzyNeural Network (F-NN) have the advantages of both fuzzy and NN system. Which means learning and optimization qualities from $\mathrm{NN}$ can be incorporated together with the human like thinking from the fuzzy system $[22,26]$.

\subsection{Generalized Dynamic Fuzzy Neural Network}

One of the Neuro Fuzzy control was established by Er and Deng [3]. Basic target of this controller was to avoid the obstacles during path planning. Architecture of the Neuro fuzzy controller is provided in Figure 4.

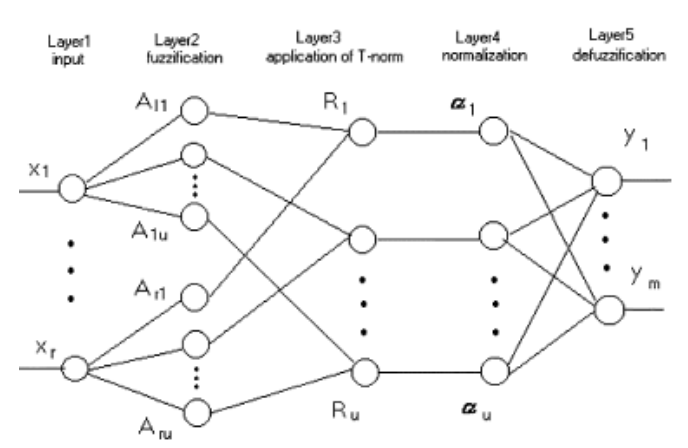

Figure 4. Generalized dynamic fuzzy neural network [3].

For the above mentioned structure, there are five layers being used in total. $\mathrm{X}$ are considered as input variables. Whereas $A, R$ and $\alpha$ are being solved with the help of Gaussian functions to provide the layer 5 .

Furthermore, with the help of supervised learning based on Generalized Dynamic Fuzzy Neural Network (GD-FNN) results were generated. One of the interesting facts of GD-FNN that they start the system without any fuzzy rules. After the learning of system fuzzy rules are either removed or re used as per their importance in the system. Due to this feature not only the parameters of the given environments can be adjusted but structure can also adapt its self.

This methodology was implemented on the simulation version of Khepera. Performance comparison of controller with and without re adaption is provided in Figure 5.

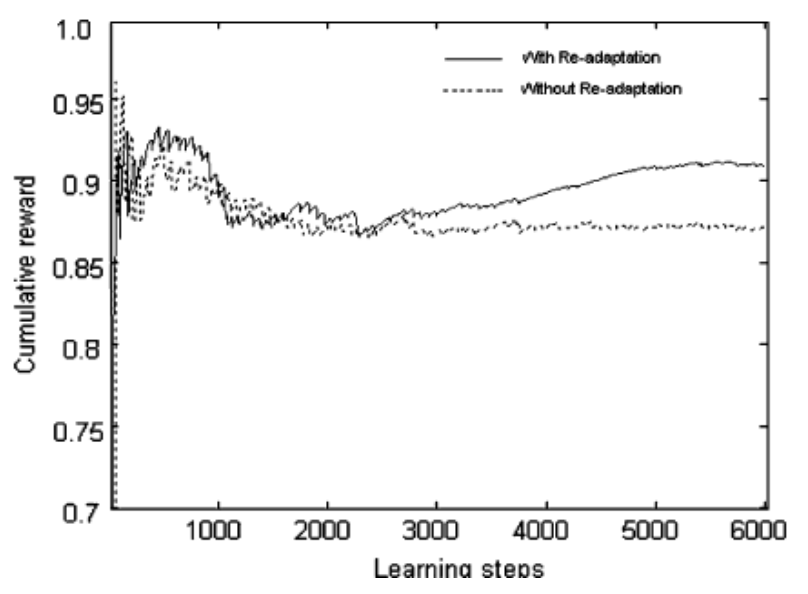

Figure 5. Performance comparison with and without reinforcement learning [3].

In this particular system its clear from the performance results that in the beginning it may look like that controller without reinforcement is behaving better than the one with the learning capability, but after some time it gradually improves and by the end it becomes superior than the controller without learning capabilities [3]. 


\subsection{Dynamic Petri Recurrent Fuzzy Neural Network}

In 2009 another algorithm was established by Wai and Liu [23] for path tracking of no holonomic Mobile Robot. It was designed to decrease the burden of computational time [23].

Dynamic Petri Recurrent Fuzzy Neural Network (DPRFNN) is basically combination of Petri net (PN) and Recurrent Fuzzy Neural Network (RFNN). PN is a tool to model and run the analysis for a number of engineering systems. It can also be used graphic tool to explain the system. In PN dynamical and concurrent system activities are simulated by the usage of tokens. State equation can also be defined with the help of PN [5].

In the beginning Wai and Liu [23] combined together the concept of PN and FNN to create PFNN. Then a system was designed by Wai and Liu [23] to include PN in RFNN to create DPRFNN.

This was done to integrate and enhance the merits of both PFNN and RFNN.

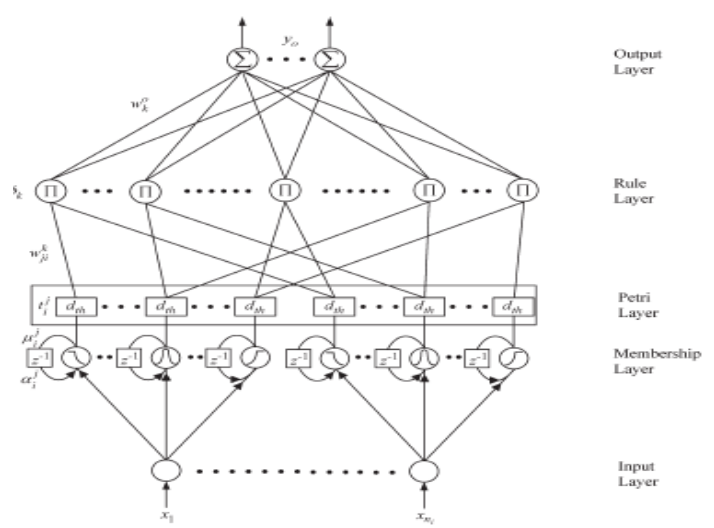

Figure 6. Dynamic petri recurrent fuzzy neural network [23].

In the structure given in Figure 6. Layer 1 is input, and it transmits the variables directly to next layer. Layer 2 is a membership layer. Layer 3 is Petri layer. Layer 4 is rule layer which multiples the inputs and provide the results. Layer 5 is output layer and each node is responsible of generating the summation of product of rule and weight.

Simulation results of all the constituent algorithms are provided the Table 1.

Table 1. Performance Parameters for FNN,RFNN, PFNN, PRFNN, DPRFNN.

\begin{tabular}{|c|c|c|}
\hline \multirow{2}{*}{ Structure } & Results & $\begin{array}{c}\text { Square Shaped Path } \\
\text { Tracking }\end{array}$ \\
\hline \multirow{2}{*}{ FNN } & Normalized Mean Square Error & $2.47 \times 10^{-3}$ \\
\cline { 2 - 3 } & Computational Time (s) & 16.32 \\
\hline \multirow{2}{*}{ RFNN } & Normalized Mean Square Error & $1.99 \times 10^{-3}$ \\
\cline { 2 - 3 } & Computational Time (s) & 16.51 \\
\hline \multirow{2}{*}{ PFNN } & Normalized Mean Square Error & $2.95 \times 10^{-3}$ \\
\cline { 2 - 3 } & Computational Time (s) & 0.24 \\
\hline \multirow{2}{*}{ PRFNN } & Normalized Mean Square Error & $2.21 \times 10^{-3}$ \\
\cline { 2 - 3 } & Computational Time (s) & 0.67 \\
\hline \multirow{2}{*}{ DPRFNN } & Normalized Mean Square Error & $1.75 \times 10^{-3}$ \\
\cline { 2 - 3 } & Computational Time (s) & 0.76 \\
\hline
\end{tabular}

From the analyzation of simulation results of all the constituent algorithms, it can be easily deduced from the that this hybrid framework is much better than the previous versions. Computational time is little bit more as compared to other structures but there is an exponential decrease in error tabulation [23].

\subsection{EIman Fuzzy Neural Network}

Recently in 2012 another technique was adopted by Wen et al. [24]. To get better performance for the mobile robots Elman Neural Network (ENN) was added as input of fuzzy proportional derivative (PD) controller. Comparison of error in simulation was carried out between simple PD control and hybrid algorithm. From the results between two methods it can be easily concluded that ENN-Fuzzy controller works far better than the regular PD control. Error comparison for both system is given in Figure 7 [24].

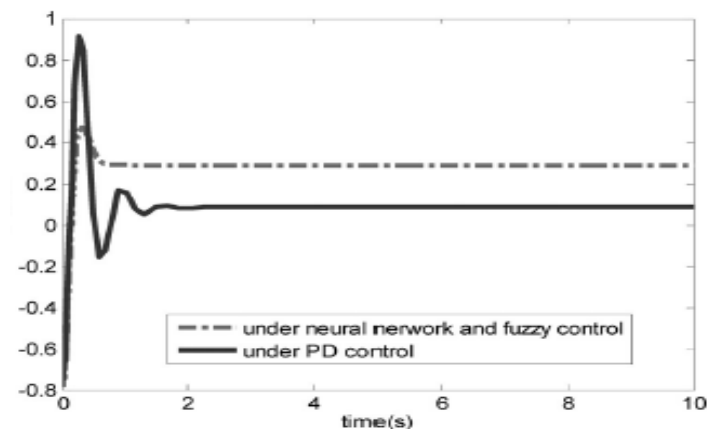

Figure 7. Error comparison of PD and fuzzy control [24].

It is clearly evident from the simulation graph that by the usage of hybrid algorithm error of the system is significantly abridged, and more stable system is achieved.

\subsection{Interval Type-2 Fuzzy Neural Network}

Initially type-1 fuzzy neural network was designed for obstacle avoidance for soccer robot system. Then in 2015 interval Type-2 fuzzy neural network (IT2FNN) for path planning of mobile robots was introduced by Kim and Chwa [9]. It was a modified design of type-1 fuzzy neural network (T1FNN). It was improved in the manner to decrease the distance and make the movement smoother. It is a three layered structure as can be seen in Figure 8. 


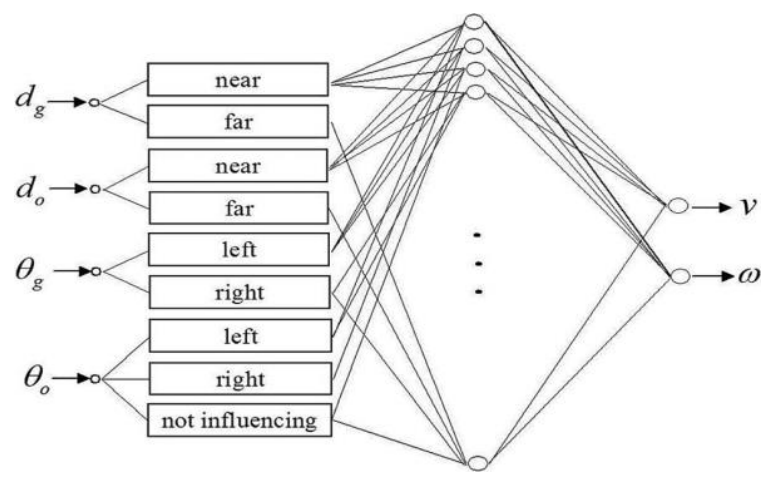

Figure 8. IT2FNN structure [9].

In this framework input layer is dependent on the distance and angle between mobile robot and obstacle. Input layer also do the fuzzification of data. Then in hidden layer membership functions are applied. Whereas in third and final layer crisp value is being determined by defuzzification of results. From the simulation results being provided in the study it can be easily deduced that IT2FNN works smoother than T1FNN. Simulation results of both frameworks are given in Figure 9. In which circle is T1FNN and trajectory path formed using square shape is for IT2FNN. Whereas * are obstacles.

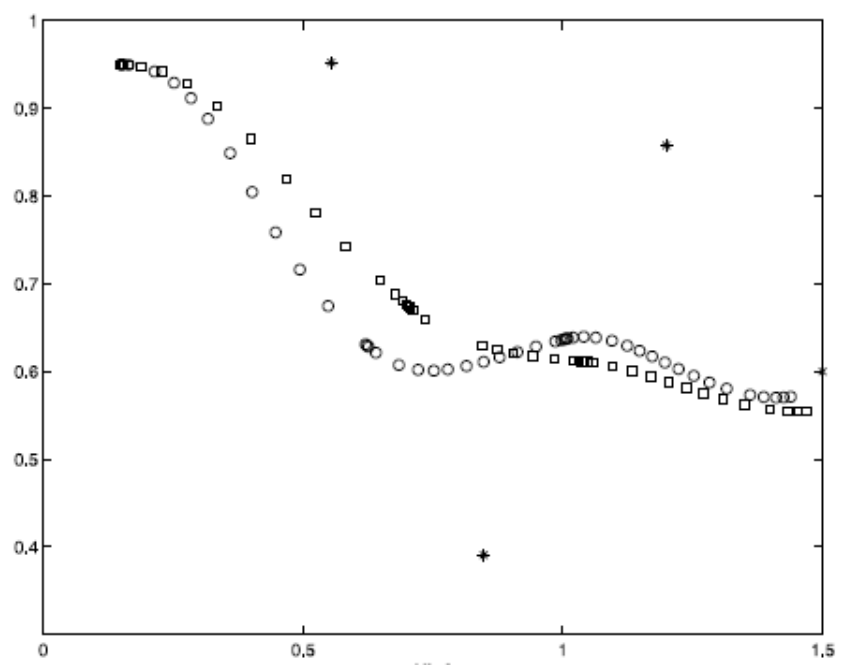

Figure 9. Trajectory Comparison [9].

Both simulation and experimental results in this scenario concluded that the proposed method of IT2FNN was much better than the previously implemented T1FNN. It didn't only decrease the travelling time but also increased the smoothness of movement during the obstacle avoidance procedure [9].

\section{Conclusions}

In this paper various techniques, which are being improvised recently in the path planning of the mobile robots are discussed. From the results it can be easily concluded that due to better accuracy and adaptiveness, hybrid approach of fuzzy logic and neural network can be very useful for the obstacle avoidance path planning. However, it remains clear that still there is a void which needs to be filled before the level of perfection is achieved.

\section{References}

[1] Allagui N., Abid D., and Derbel N., "Fuzzy PI Controller for Mobile Robot Navigation and Tracking," in Proceedings of $15^{\text {th }}$ International Multi-Conference on Systems, Signals and Devices (SSD), Hammamet, pp. 1178-1183, 2018.

[2] Emmi L. and Gonzalez-de-Santos P., "Mobile Robotics in Arable Lands: Current State and Future Trends," in Proceedings of European Conference on Mobile Robots, Paris, pp. 1-6, 2017.

[3] Er M. and Deng C., "Obstacle Avoidance of A Mobile Robot Using Hybrid Learning Approach," IEEE Transactions on Industrial Electronics, vol. 52, no. 3, pp. 898-905, 2005.

[4] Fox D., Burgard W., and Thrun S., "The Dynamic Window Approach to Collision Avoidance," IEEE Robotics and Automation Magazine, vol. 4, no. 1, pp. 23-33, 1997.

[5] Furlán F., Rubio E., Sossa H., and Ponce V., "Humanoid Robot Hierarchical Navigation Using Petri Nets and Fuzzy Logic," in Proceedings of $56^{\text {th }}$ Annual Conference of the Society of Instrument and Control Engineers of Japan (SICE), Kanazawa, pp. 1521-1526, 2017.

[6] Glasius R., Komoda A., and Gielen S., "Neural Network Dynamics for Path Planning and Obstacle Avoidance," Neural Networks, vol. 8, no. 1, pp. 125-133, 1994.

[7] Gomide F., Rocha A., and Albertos P., "Neurofuzzy Controllers," IFAC Proceedings Volumes, vol. 25, no. 25, pp. 13-26, 1992.

[8] Kermiche S., Larbi S., Abbassi H.," Fuzzy Logic Control of Robot Manipulator in The Presence of Fixed Obstacle," The International Arab Journal of Information and Technology, vol. 4, no.1, pp. 26-32, 2007.

[9] Kim C. and Chwa D., "Obstacle Avoidance Method for Wheeled Mobile Robots Using Interval Type-2 Fuzzy Neural Network," in IEEE Transactions on Fuzzy Systems, vol. 23, no. 3, pp. 677-687, 2015.

[10] Lozano-Pérz T. and Wesley M., "An Algorithm for Planning Collision- Free Paths Among Polyhedral Obstacles," Commun. ACM, vol. 22, no. 10, pp. 560-570, 1979.

[11] Mirza N., "Comparison of Artificial Neural Networks based on Controllers for Biped Robots," TEM Journal, vol. 8, no. 4, pp. 12721276, 2019.

[12] Mirza N., "Application of Fuzzy Neural Networks in Robotic Path Planning," The 
International Arab Conference on Information Technology, Al Ain, pp. 58-62, 2019.

[13] Naik K. and Gupta C., "Performance Comparison of Type-1 and Type-2 Fuzzy Logic Systems," in Proceedings of $4^{\text {th }}$ International Conference on Signal Processing, Computing and Control, Solan, pp. 72-76, 2017.

[14] Nejattukenmez. (2017, January 22). What is Fuzzy Logic?

from https://nejattukenmez.wordpress.com/2016/05/24/b ulanik-mantik-nedir/, Last Visited, 2020.

[15] Pandey A., Sonkar R. K., Pandey K. K. and Parhi D. R., "Path Planning Navigation Of Mobile Robot With Obstacles Avoidance Using Fuzzy Logic Controller," in Proceedings of $8^{\text {th }}$ International Conference on Intelligent Systems and Control, Coimbatore, pp. 39-41, 2014.

[16] Qu H., Yang S., Willms A. and Yi Z., "Real-Time Robot Path Planning Based on a Modified PulseCoupled Neural Network Model," IEEE Transactions on Neural Networks, vol. 20, no. 11, pp. 1724-1739, 2009.

[17] Sandeep B. and Supriya P., "Analysis of Fuzzy Rules For Robot Path Planning," in Proceedings International Conference on Advances in Computing, Communications and Informatics, Jaipur, pp. 309-314, 2016.

[18] Sangeetha V. and Ravichandran K., "A Modified Fuzzy A* Based Inference System for Path Planning in an Unknown Environment," in Proceedings $2^{\text {nd }}$ International Conference on Trends in Electronics and Informatics, Tirunelveli, pp. 181-186, 2018.

[19] Smith Steven W., "The Scientist and Engineer's Guide to Digital Signal Processing" The Scientist and Engineer's Guide to Digital Signal Processing. [Online].Available:

http://www.dspguide.com/ch26/2.htm, Last Visited, 2020.

[20] Tan Ai R. and Dadios E., "Neuro-Fuzzy Mobile Robot Navigation," in Proceedings $10^{\text {th }}$ International Conference on Humanoid, Nanotechnology, Information Technology, Communication and Control, Environment and Management, Baguio City, pp. 1-6, 2018.

[21] Tutorialspoint.com, "Artificial Intelligence Fuzzy Logic Systems," www.tutorialspoint.com. [Online]. Available: https://www.tutorialspoint.com/artificial_intelligen ce/artificial_intelligence_fuzzy_logic_systems.htm, Last Visited, 2020.

[22] Ullah Z., Xu Z., Zhang L., Zhang L., and Ullah W., "RL and ANN Based Modular Path Planning Controller for Resource-Constrained Robots in the Indoor Complex Dynamic Environment," IEEE Access, vol. 6, pp. 74557-74568, 2018.

[23] Wai R. and Liu C., "Design of Dynamic Petri Recurrent Fuzzy Neural Network and its
Application to Path-Tracking Control of Nonholomonic Mobile Robot," IEEE Transactions on Industrial Electronics, vol. 56, no. 7, pp. 2667-2683, 2009.

[24] Wen S., Zheng W., Zhu J., Li X., and Chen S., "Elman Fuzzy Adaptive Control for Obstacle Avoidance of Mobile Robots Using Hybrid Force/Position Incorporation," IEEE Transactions on Systems, Man, and Cybernetics, Part $C$ (Applications and Reviews), vol. 42, no. 4, pp. 603-608, 2012.

[25] Yang S. and Luo C., "A Neural Network Approach to Complete Coverage Path Planning," IEEE Transactions on Systems, Man, and Cybernetics, Part B (Cybernetics), vol. 34, no. 1, pp. 718-724, 2004.

[26] Yudha H. M., Dewi T., Hasana N., Risma P., Oktarini Y., and Kartini S., "Performance Comparison of Fuzzy Logic and Neural Network Design for Mobile Robot Navigation," in Proceedings of International Conference on Electrical Engineering and Computer Science, Batam Island, pp. 79-84, 2019.

[27] Zennir Y. and Allou S., "Comparison of PID and Fuzzy Controller for Path Tracking Control of Autonomous Electrical Vehicles," in Proceedings of International Conference on Electrical Sciences and Technologies in Maghreb, Algiers, pp. 1-6, 2018.

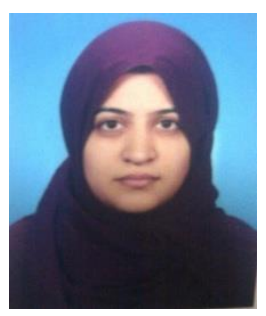

Nada Mirza currently serves as an Instructor in the College of Engineering at $\mathrm{Al}$ Ain University (AAU), UAE. Ms. Nada received her BE and MS degrees in Mechatronics Engineering from College of Electrical and Mechanical Engineering, National University of Sciences \& Technology, Pakistan. Her post-graduate research was mostly focused on the application of artificial intelligence, robotics and wireless monitoring of renewable energy systems. Her research is currently focused on cognitive radio networks, MIMO radio channel characterization for microwave and wireless body area networks, alternative sampling methodologies for the sampling of multidimensional signals and adaptive signal processing techniques, and machine learning. 